\title{
Neonatal Choanal Atresia: A Series of 13 Cases
}

\author{
F. Hadid, M. Hakimi, O. Benhommad, Y. Rochdi, and A. Raji
}

\section{ABSTRACT}

Choanal atresia is a congenital disease that Is manifested at birth when it is bilateral. Unilateral forms can be observed later in life as they are usually well tolerated. Choanal atresia's frequency is difficult to estimate. It is estimated to be 1 case per 5000 to 8000 births [1]-[3]. Our work is a retrospective study of the cases of choanal atresia that were managed in the ENT department of the Mohamed VI University Hospital during a period of eleven years from January 2010 to December 2020. We identified 13 cases of choanal atresia. The aim of this work is to analyze the elements of the diagnosis, the indications and the surgical techniques as well as our results of the endonasal endoscopic approach in order to identify a therapeutic strategy. The average age of our patients was 21 days, with extremes ranging from 1 day to 28 days. The maximum number of cases was observed in newborns during the first week of life, with a slight female predominance of $7 / 6$. The sex ratio was 0,85 . In our series, the delay was most often short with extremes ranging from day 1 of life to day 22 . There were 9 bilateral imperforations $(69.23 \%)$, and 4 unilateral imperforations $(30.77 \%), 2$ on the right side $(50 \%)$ and 2 on the left side $(50 \%)$. The diagnosis of choanal atresia was confirmed by computed tomography (CT) and nasal endoscopy. All our patients underwent surgical treatment by endonasal endoscopic approach with calibration with a favorable evolution. Through this study, we concluded that the frequent association with other anomalies requires a clinical and paraclinical evaluation and a long-term follow-up. Nasal endoscopy and CT scan have completely revolutionized the diagnostic and therapeutic approaches of choanal atresia, and the endoscopic endonasal approach remains the best therapeutic choice.

Keywords: atresia- choanal- Endoscopic endonasal approach.

Submitted : May 02, 2021

Published : May 25, 2021

ISSN: 2593-8339

DOI: $10.24018 /$ ejmed.2021.3.3.851

\section{F. Hadid*}

Ear, Nose and Throat Department, Mohammed VI University Hospital, Marrakesh, Morocco.

(e-mail: fz.hadid@gmail.com)

M. Hakimi

Ear, Nose and Throat Department,

Mohammed VI University Hospital,

Marrakesh, Morocco.

O. Benhommad

Ear, Nose and Throat Department, Mohammed VI University Hospital, Marrakesh, Morocco.

Y. Rochdi

Ear, Nose and Throat Department,

Mohammed VI University Hospital, Marrakesh, Morocco.

A. Raji

Ear, Nose and Throat Department, Mohammed VI University Hospital, Marrakesh, Morocco.

*Corresponding Author

\section{INTRODUCTION}

Choanal atresia is a relatively rare disease ( 1 in 5000 to 8000 births) [1]-[3]. First described in 1755, then defined anatomically by Otto in 1829 by the abnormal presence of an obstructive tissue preventing any communication between the nasal cavity and the nasopharynx. It can be unilateral or bilateral, partial, or complete, osseous, membranous, or mixed [4]. The first surgical cure was performed in 1853 by Emmert, who had successfully performed endonasal surgery on a 7-year-old child [5]. The diagnosis, primarily clinical, has been considerably facilitated by endoscopy. Currently, computed tomography (CT) is the procedure of choice in the evaluation of choanal atresia [6], [7]. The problem with this disease is the therapeutic management and recurrence is the most frequent complication.

\section{MATERIALS AND METHODS}

Our work is a retrospective study of the cases of choanal atresia that were managed in the ENT department of the Mohamed VI University Hospital during a period from
January 2010 to December 2020. We identified 13 cases of choanal atresia. This is a retrospective study of all patients with choanal atresia hospitalized in the neonatal intensive care unit.

\section{RESULTS}

The average age of our patients was 21 days, with extremes ranging from 1 day to 28 days. The maximum number of cases was observed in newborns during the first week of life, with a slight female predominance of $7 / 6$. The sex ratio was 0.85 . In our series, the delay was most often short with extremes ranging from Day 1 of life to Day 22 of life. The circumstances of discovery differ depending on if atresia is unilateral or bilateral. The bilateral form was generally revealed by signs of neonatal respiratory distress either moderate (6 cases) or severe (3 cases). ENT examination showed non-permeable nasal cavities on catheterization with a flexible nasal endoscope. There were 9 bilateral imperforations $(69.23 \%)$, and 4 unilateral imperforations $(30.77 \%), 2$ on the right side $(50 \%)$ and 2 on the left side (50\%). Nasal endoscopy was used to support the diagnosis of all our patients and to clarify if they had complete or partial atresia (Fig. 1). 


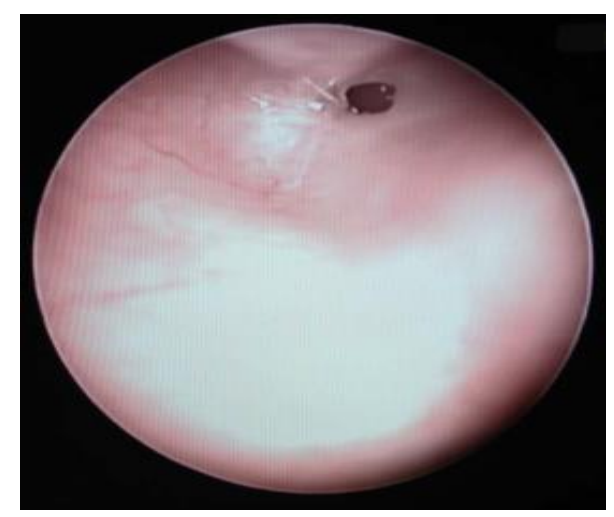

Fig.1. Endoscopic view of a choanal atresia.

The osseous, membranous, or mixed nature of the choanal atresia is determined by CT scan or intraoperatively. In our series, the atresia was osseous in 3 cases (23\%), membranous in 5 cases $(38.5 \%)$, and mixed in 5 cases $(38.5 \%)$ (Fig. 2, 3, 4).

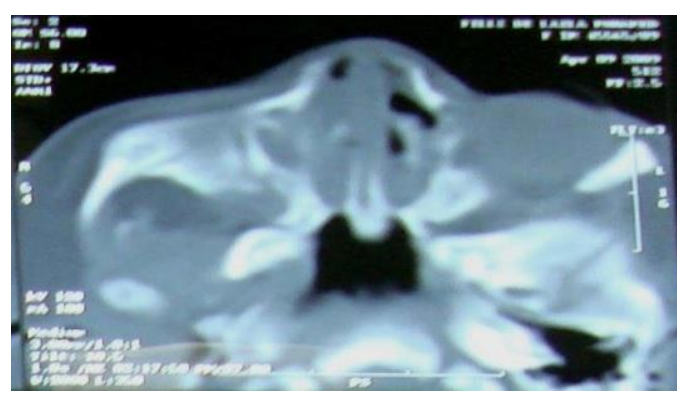

Fig. 2. Scannographic aspect of osseous choanal atresia.

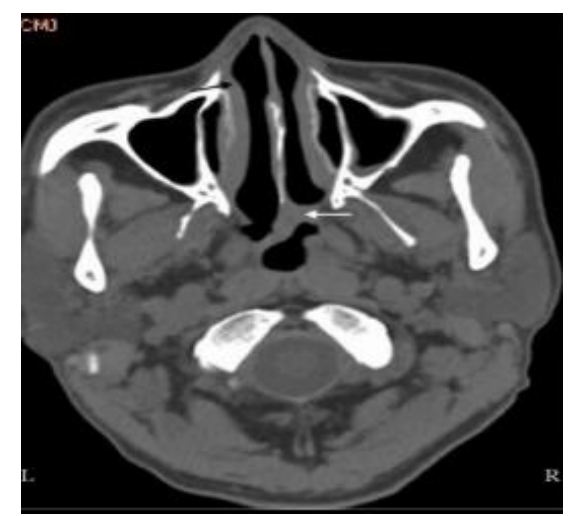

Fig. 3. Scannographic aspect of membranous choanal atresia.

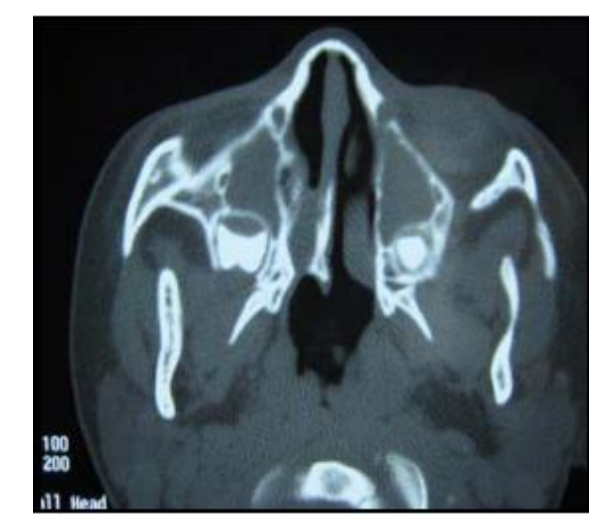

Fig. 4. Scannographic aspect of mixed choanal atresia.

Five newborns had associated malformations, such as hydrocele with myxedema, atrial septal defect with ostium secondum, micro penis, reducible umbilical hernia, and 2 newborns with cleft lip and palate.
In the case of bilateral choanal atresia, the first therapeutic step is to open the airway, keep the newborn's mouth open with a Guedel or Mayo cannula, and place an oral gastric tube. In the case of bilateral choanal atresia, patients were operated on as an emergency. In the case of unilateral choanal atresia, the patients were operated on in a delayed way. Only one approach was adopted in our patients (the endoscopic endonasal approach). The surgical technique consists of an inspection of the two nasal cavities, to identify the atretic plaque on both sides, to localize the floor of the choana where the opening of the atretic plate should begin. The opening of the atretic plate with a scalpel with progressive erosion of the bone wall. Choanal enlargement was considered satisfactory when two ageappropriate nasotracheal intubation tubes could be passed simultaneously and without friction on both sides. Placement of a nasal catheter for calibration and then fixation of the assembly at the vestibular level (Fig. 5). Calibration lasted between 6 and 12 weeks with an average of 8 weeks. The evolution was favorable in all 12 of our newborns (Improvement on the respiratory level, absence of dyspnea during feedings). One baby died on the 3rd postoperative day due to a neonatal infection of pulmonary origin. Two newborns presented postoperative respiratory disorders following the fall of the assembly, they were taken back surgically by endoscopic endonasal approach with a good evolution.

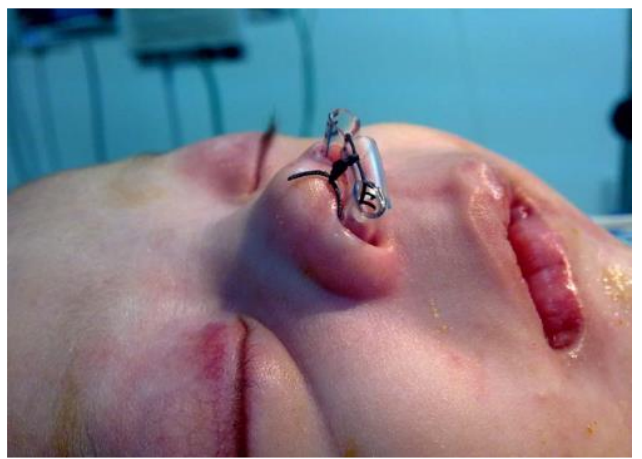

Fig. 5. Calibration in a newborn operated for choanal atresia (side view).

\section{DISCUSSION}

Choanal atresia is a congenital disease that is manifested at birth when it is bilateral. Unilateral forms can be observed later in life as they are usually well tolerated. The frequency of choanal atresia is difficult to estimate. The frequency is estimated to be 1 case per 5000 to 8000 births [1]-[3], in our series we counted 13 cases over 11 years. CANNONI has reported in his series 25 cases of choanal atresia of which 7 were diagnosed at birth [3]. A clear female predominance is found in most of the series but no ethiopathogenic reason has been found and remains a simple epidemiological observation [2]. The unilateral form seems to be more frequently observed than the bilateral form. According to some studies, the results vary from 60 to $64 \%$ of unilateral involvement versus 40 to $36 \%$ of bilateral involvement. Our results were different from the ones found in the literature since we found more bilateral than unilateral forms, which can be explained by the fact that unilateral atresia are well tolerated. The goal of treatment of choanal atresia is the 
restoration of a normal airway, stable over time. Surgical treatment involves several techniques but the transpalatal and transnasal approaches are the most commonly practiced [4], [5]. The indications depend essentially on the age of the patient, the uni or bilaterality, the nature of the atresia, the existence of associated anomalies, the specific risks of each technique and the possible previous surgical failure. In case of bilateral choanal atresia, all authors agree on the need to ensure airway clearance by placing a Mayo or Guedel cannula. In exceptional cases, orotracheal intubation is indicated. Tracheostomy is reserved for forms with craniofacial or cardiorespiratory malformations [1], [6], [8]. The endoscopic approach has significantly developed since the first description by Stankiewitz. It has benefited from the appearance of modern instrumentation, the burrs and the micro-debrider [1], [7], this approach was practiced in all our patients. It has the advantage of offering good visualization; allowing extensive resection; a short procedure with low morbidity that can be offered from birth to bilateral choanalatresias, whether mixed or osseous. The choanal calibration recommended in all the literature, recent or old, is an essential part of the intervention for these authors [1]. The duration of calibration can be reduced thanks to new surgical techniques (use of mucosal flaps [9], [10] and laser [7]).

\section{CONCLUSION}

We concluded through this study that nasal endoscopy and computerized tomography have completely revolutionized the diagnostic and therapeutic approaches of choanal atresia, and that the endoscopic endonasal approach continues to be the best therapeutic choice.

\section{REFERENCES}

[1] N.R. Friedman, R.B. Mitchell, C.M. Bailey, et al., Management and outcome of choanal atresia correction, Int. J. Pediatr. Otorhinolaryngol. 52 (2000) 45-51.

[2] J.L. Keller, A. Kacker, Choanal Atresia, CHARGE Association, and Congenital Nasal Stenosis, Otolaryngol. Clin. North Am. 33 (6) $(8$ December 2000) 1343-1351.

[3] Cannoni M, Thomassin JM, Meyen A. L'imperforationchoanale. Topographie de la malformation et intérêt de la voie transpalatine. J fr, oto- rhino-laryngol $1983 ; 32$ P:289-93.

[4] V. Sinha, D. Gurnani, N.R. Modi, D.A. Barot, Choanal atresia: surgical managementbyHegar's dilators, Indian J. Otolaryngol. Head Neck Surg. 66 (3) (2014) 272-275(Sep).

[5] J.D. Ramsden, P. Campisi, V. Forte, Choanal atresia and choanal stenosis, Otolaryngol. Clin. N. Am. 42 (2009) 339-352.

[6] H. Hajri, S. Mannoubi, N. Mathlouthi, N. Kaffel, M. Marrakchi, H. Kooli, M. Ferjaoui, Imperforation choanale aspects cliniques, approche therapeutique j. Tun orl - $\mathrm{n}^{\circ} 17 \mathrm{~d}$ ecembre 2006, P. 30-34 11.

[7] Bart D. Vanzieleghem, Marc M. Lemmerling, Hubert F. Vermeersch, Paul Govaert, et al. Imaging Studies in the Diagnostic Workup of Neonatal Nasal Obstruction. Journal of Computer Assisted Tomography; Vol. 25, No. 4, 2001; p: 540-549.

[8] Khalifa N, Calas V, Tremelet L, Cave J.P. L'obdtruction nasale chez l'enfant. Rev. de la société ORL de Tarn-et-Garonne; octobre 2007 $n^{\circ} 03$.

[9] Cedin Ac, Fujitar, Cruz Olm. Endoscopic transeptal surgery for choanal atresia with a stentless folded-over-flap technique. Otolaryngology-head and neck surgery, 2006, 135, P:693-8.

[10] Pasquini E, Sciarretta V, Saggese D, Cantaroni C, Macri G, Farneti G. Endoscopic treatment of congenital choanal atresia. International Journal of Pediatric Otorhinolaryngology (2003) 67, 271_276. 